\title{
Hypertone Kochsalzlösung bei Kleinkindern ohne Wirkung?
}

\section{Die Inhalation einer hypertonen Kochsalzlösung kann die Flüssigkeit des Atemwegssekrets und die mukozilliäre Clearance bei Mukoviszidose-Patienten verbessern. Bei älteren Kindern und Erwachsenen reduziert diese Therapie pulmonale Exazerbationen. Ob dies auch für Kinder unter 6 Jahre zutrifft, haben nun M. Rosenfeld et al. untersucht.}

JAMA 2012; 307: 2269-2277

An der randomisierten, doppelverblindeten Multicenterstudie „Infant Study of Inhaled Saline in Cystic Fibrosis“ (ISIS) nahmen Kinder im Alter zwischen 4 und 60 Monaten mit bekannter MukoviszidoseDiagnose teil. Sie inhalierten über 48 Wochen 2-mal täglich entweder 7\%ige hypertone Kochsalzlösung (aktive Therapie) oder 0,9\%ige isotonische Kochsalzlösung (Kontrollgruppe). Die Patienten wurden bei Aufnahme bzw. Randomisierung sowie nach 4, 12, 24, 36 und 48 Wochen untersucht. Die Familien wurden 2 Wochen nach Studienaufnahme, danach vierteljährlich kontaktiert. Dabei wurden Informationen per Fragebögen erhoben. Primärer Endpunkt war die Rate pulmonaler Exazerbationen, die eine Antibiotikabehandlung erforderten. Sekundäre Wirksamkeitsparameter waren Gewicht, Größe, Atemfrequenz in Ruhe, Sauerstoffsättigung (bei Raumluft) und Husten. Die Autoren gingen von einer Exazerbationsrate in der Kontrollgruppe von 2,22 Ereignissen/Jahr aus.
Insgesamt konnten 321 Patienten randomisiert werden: 158 auf hypertone bzw. 163 auf isotone Kochsalzlösung. Die Kinder waren im Mittel 2,2 bzw. 2,3 Jahre alt. In der aktiven Therapiegruppe dauerte die durchschnittliche Studienteilnahme 47 und in der Kontrollgruppe 46 Wochen. Pro Jahr und Patient kam es sowohl unter aktiver Therapie als auch in der Kontrollgruppe zu 2,3 pulmonalen Exazerbationen. Das Verhältnis durchschnittlicher schwerer Atemwegsinfektionen von hypertoner zu isotoner Kochsalzlösung betrug 0,97 (95\%-Konfidenzintervall [KI], 0,83-1,13) und die Hazard Ratio für die Zeit bis zur ersten Exazerbation 0,94 (95\%-KI, 0,74-1,21).

Unter den Teilnehmern, die Exazerbationen erlitten, war eine Antibiotikatherapie an durchschnittlich 60 Tagen (aktive Therapie) bzw. 52 Tagen (Kontrollgruppe) notwendig. Bei den sekundären Endpunkten sowie in Lungenfunktionstests

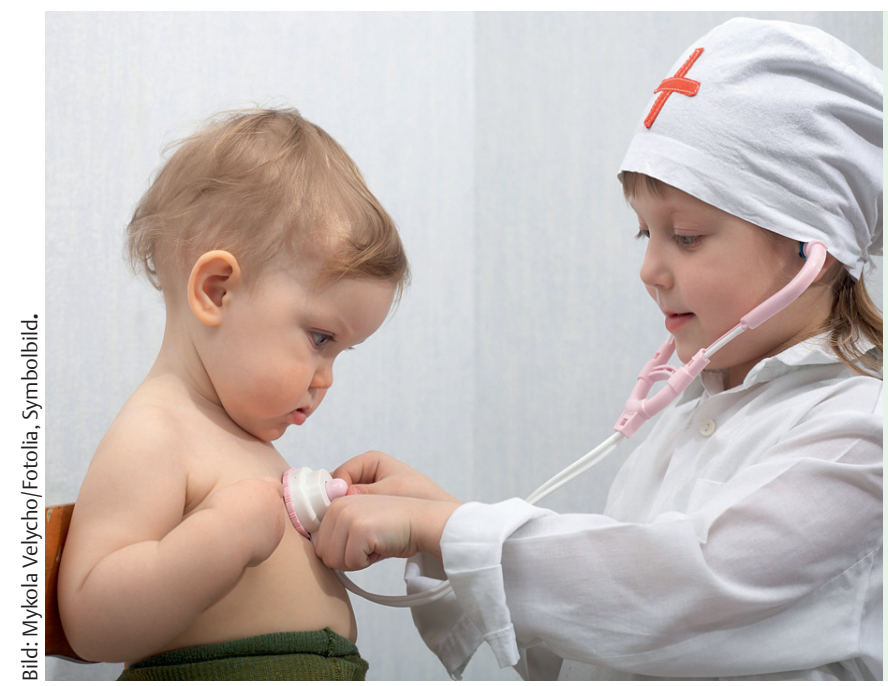

(in einer Subgruppenanalyse) ergaben sich keine deutlichen Unterschiede zwischen den Behandlungsgruppen. Die Therapieadhärenz erreichte insgesamt $75 \%$. Die häufigste Nebenwirkung mit mittlerer bis schwerer Ausprägung war Husten bei 39\% der Patienten unter hypertoner Kochsalzlösung bzw. 38\% der Kontrollpatienten.

Fazit
Mukoviszidose-Patienten unter 6 Jah-
ren profitieren in Bezug auf die Rate
pulmonaler Exazerbationen nach An-
gaben der Autoren nicht von der Inha-
lation hypertoner Kochsalzlösung. In
der ISIS-Studie hat diese Behandlung
im Vergleich zu isotoner Kochsalzlö-
sung die Zahl schwerer Atemwegsin-
fektionen nicht reduzieren können.

Matthias Manych, Berlin

\section{Ausschreibung}

\section{Forschungspreis der Sarkoidose Stiftung}

Die gemeinnützige Sarkoidose Stiftung schreibt anläßlich ihres 10-jährigen Jubiläums für 2013 die finanzielle Förderung von Forschungsvorhaben zur Sarkoidose und/oder anderer granulomatöser $\mathrm{Er}$ krankungen aus. Es sollen Projekte gefördert werden, die sich der Grundlagenforschung oder der klinisch orientierten Erforschung dieser Krankheitsbilder widmen. Es können Personal-, Sach- und Reisekosten bis zu einer Gesamtsumme von $10000 €$ gefördert werden, die in der Regel innerhalb eines Jahres verausgabt werden sollen.

An der ISIS-Studie nahmen Kinder zwischen 4 Monaten und 5 Jahren mit einer diagnostizierten Mukoviszidose teil. Die Inhalation von hypertoner Kochsalzlösung reduzierte im Vergleich zur Kontrollgruppe jedoch nicht die Exazerbationsrate.
Der Antrag besteht aus einer auch für Fachfremde verständlichen Projektskizze auf nicht mehr als 10 Seiten, einer einseitigen Zusammenfassung des Forschungsthemas, dem Lebenslauf, dem Verzeichnis bisheriger Veröffentlichungen und ggf. bereits abgewickelter Forschungsprojekte. Die Bewerber werden gebeten, ihren Antrag bis zum 31. Juli 2013 bei der Sarkoidose Stiftung via E-Mail an Forschung@ Sarkoidose-Stiftung.de einzureichen.

Mitteilung der Sarkoidose Stiftung, Meerbusch 